\title{
A Computer Model for Teaching the Dynamic Behavior of AC Contactors
}

\author{
Jordi-Roger Riba Ruiz, Antonio Garcia Espinosa, Member, IEEE, and Luis Romeral, Member, IEEE
}

\begin{abstract}
-ac-powered contactors are extensively used in industry in applications such as automatic electrical devices, motor starters, and heaters. In this work, a practical session that allows students to model and simulate the dynamic behavior of ac-powered electromechanical contactors is presented. Simulation is carried out using a rigorous parametric model of the ac contactor that avoids simplification assumptions and is thoroughly explained. The goal of this practical is to introduce students to the topic of dynamic simulation of real devices. It covers both the transient and the steady-state response of the electromechanical system under study. The proposed methodology is flexible and not particularly time-consuming, and it allows the students easily to change the electromechanical constants of the contactor they are studying. The results of the simulations were compared with experimental data acquired by the students; a close similarity between real and simulated data was observed. The Technical University of Catalonia (UPC), Spain, has incorporated the simulation methodology proposed in this paper in a practical session of an electrical engineering course.
\end{abstract}

Index Terms-Computer-assisted learning, dynamic modeling, education, electromechanical contactor, shading rings, simulation.

\section{INTRODUCTION}

$\mathbf{E}$ LECTROMECHANICAL contactors have been extensively used in industry for many decades; over $80 \%$ of them are equipped with ac-powered coils [1]. Contactors are used in applications that require circuit making and breaking, such as electrical automatisms, circuits containing motor starters, heaters, and lights. One of their drawbacks is the mechanical movement of some of their parts and the wear that this causes. The mechanical movement also causes the contacts to impact during closure, leading to contact bounce. This undesirable phenomenon, due to the reopening of the contacts during closure, reduces the estimated life of the contactors because they degrade [2], [3] and overheat.

The coil of the contactor can be powered by an ac or a dc supply. dc-powered coils generate a constant magnetic field that guarantees an appropriate closing of the contacts. ac-powered

Manuscript received June 10, 2008. First published June 30, 2009; current version published May 05, 2010.

J.-R. Riba Ruiz is with the EUETII and the Electrical Engineering Department, Universitat Politècnica de Catalunya (UPC), Igualada 08700, Spain (e-mail: jordi@euetii.upc.edu).

A. Garcia Espinosa is with the Electrical Engineering Department, Universitat Politècnica de Catalunya (UPC), Terrassa 08222, Spain (e-mail: garciae@ee.upc.edu).

L. Romeral is with the Electronic Engineering Department, Universitat Politècnica de Catalunya (UPC), Terrassa 08222, Spain (e-mail: romeral@eel.upc.edu).

Digital Object Identifier 10.1109/TE.2009.2014153 coils, on the other hand, generate sinusoidal magnetic fields. These alternating magnetic fields produce a force that drops to zero twice in each cycle $(50 / 60 \mathrm{~Hz})$, giving rise to an undesired chatter or vibration of the contacts. This effect can be eliminated by placing a metal shading ring in the contactor core to act as a secondary winding. The current flowing through this ring generates a magnetic field that is out of phase with that generated by the contactor coil. This setup produces a resultant force that does not drop to zero, thus eliminating the vibrations.

Most electrical engineering courses are still based on wellestablished steady-state models of electrical machinery. These models, however, cannot describe the transient behavior of a machine. Today, with the extensive use of low-cost computers, the dynamic modeling and simulation of electromechanical systems have become essential tools for design verification, operation studies, and design and behavior improvement. The modeling of real devices has taken on great importance in engineering courses [4].

The aim of the work presented here is to introduce this methodology to students on electrical engineering courses by modeling and simulating the dynamic behavior of an ac-powered electromechanical contactor. This methodology is very useful for students because it involves topics such as modeling electromechanical actuators, dynamic simulation, establishing and comprehending physical laws, solving electrical and magnetic circuits, and interpreting electromagnetic coupling problems. The model proposed in this work allows the students to compute and show electrical, magnetic, and mechanical magnitudes - some of which are difficult to measure-on a graph. It has two distinct advantages: The simulations are not particularly time-consuming (students take a few seconds to solve the equations), and the mechanical and electrical parameters that characterize each contactor can be modified easily.

In this research, Matlab-Simulink, a software platform specialized in numerical computing that is widely used in engineering courses, has been used [5]-[9].

\section{Course Structure AND DetAils}

The Electrotechnology course is taught at the Department of Electrical Engineering of the Technical University of Catalonia (UPC), Spain. The laboratory practical presented in this paper was taught to fifth-semester undergraduate students taking the Electrotechnology course at the School of Industrial and Aeronautical Engineering of Terrassa (ETSEIAT), Spain. The practical consists of two sessions lasting $2 \mathrm{~h}$ each. Electrotechnology has been a very successful course, and most of the students have expressed their satisfaction with the proposed learning methodology and the content. In the practical sessions held in the laboratory of the school, students write the code program under the 
supervision of the instructors. The source code is programmed using the Matlab-Simulink environment. Note that Electrotechnology students in the course had worked with Matlab-Simulink before.

The students on the Electrotechnology course had previously taken the following courses, whose contents are related to those needed in the proposed practical:

- Statics and Dynamics (first semester)

- Electricity and Magnetism (third semester)

- Theory of Circuits and Systems (fourth semester)

- Fundamentals of Electrical Engineering (fifth semester).

\section{A. Course Syllabus}

At the School of Industrial and Aeronautical Engineering of Terrassa, the Electrotechnology course is taught over a 15-week semester. The classes meet for four 1-h sessions every week. Moreover, every two weeks, groups of three students attend a practical session that lasts a minimum of $2 \mathrm{~h}$, depending on how well the students do in their assignments. On average, students dedicate 4 extra hours of work to finishing the calculations, simulations, and reports related to each practical session. The laboratory reports are submitted at the following laboratory session. The laboratory is divided into two parts: one contains workbenches, electrical machines and measuring instruments, and the other is a PC room.

The basic content of the Electrotechnology course is given below. (The theoretical concepts related to contactors and electromechanical conversion principles are studied in Module 2.)

- Module 1. Three-phase systems

- Module 2. Fundamentals of electrical machines

- Module 3. Transformers

- Module 4. Induction machines

- Module 5. Direct current machines.

\section{Magnetic CiRCUIT Modeling}

In this work, an ac-powered contactor is dealt with, a sketch of which is shown in Fig. 1. Its components are the main coil and the shading rings, the fixed core, the movable core (also known as the armature), the air gaps, the return springs, a holder attached to the top of the armature, and three sets of moving contacts that are guided by the contact holder. Each contact is preloaded by its respective phase spring.

To determine the equations that govern the dynamic behavior of the contactor, a magnetic model is needed. The following symbols are used:

$\begin{array}{ll}d_{A \_0} & \text { initial precompression of the return spring } \\ d_{A} & \text { resting position of the movable core } \\ x, \dot{x}, \ddot{x} & \begin{array}{l}\text { instantaneous position, velocity and } \\ \text { acceleration of the movable core }\end{array} \\ m_{A} & \begin{array}{l}\text { mass of the movable core } \\ d_{C \_0}\end{array} \\ & \begin{array}{l}\text { initial precompression of the three phase } \\ \text { springs }\end{array} \\ d_{C} & \text { resting position of the three movable contacts }\end{array}$

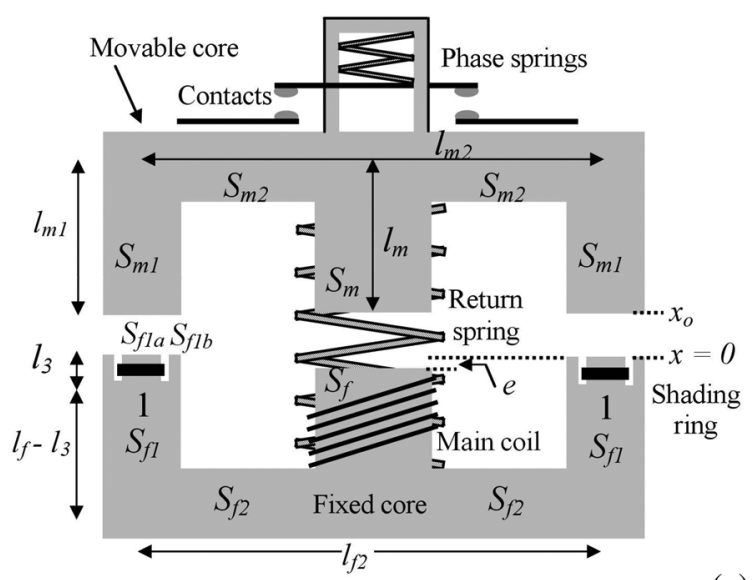

(a)

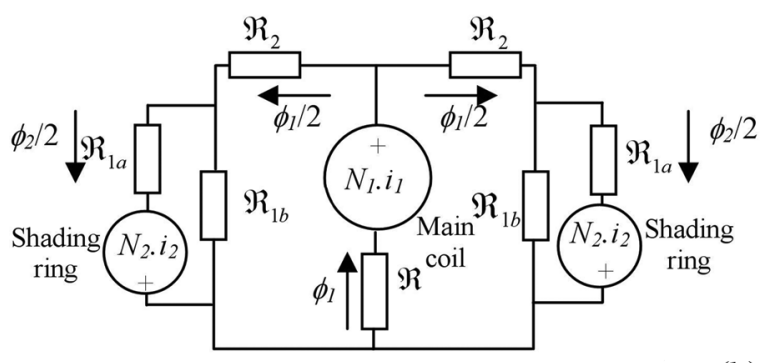

(b)

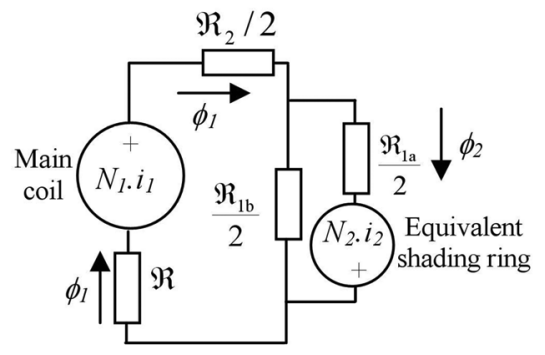

Fig. 1. (a) A sketch of the electromagnetic contactor, (b) the magnetic circuit, and (c) the equivalent magnetic circuit.

$x_{C}, \dot{x}_{C}, \ddot{x}_{C}$ instantaneous position, velocity and acceleration of the three movable contacts

$3 m_{C} \quad$ resultant mass of the three movable contacts

$k_{1}$ elastic constant of the return spring

$3 k_{2} \quad$ equivalent elastic constant of the three phase springs

$b$

friction constant

$\varepsilon$

restitution coefficient

$F_{k_{1}}$ force of the return spring

$F_{3 k_{2}}$ resultant force of the three phase springs

$F_{f} \quad$ force of friction

F fringing factor $>1$

$R_{1} \quad$ electrical resistance of the main coil $R_{2} \quad$ electrical resistance of each shading ring

$N_{1} \quad$ number of turns in the main coil 


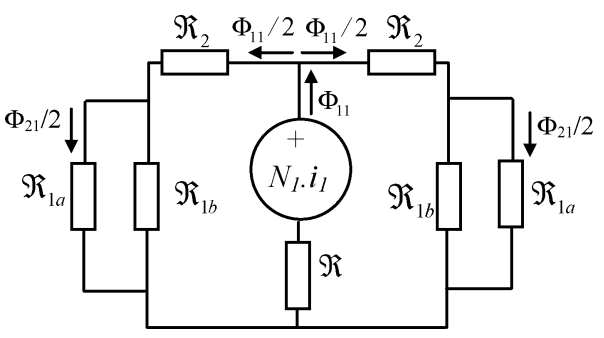

Fig. 2. Equivalent magnetic circuit for calculating $\Phi_{11}, L_{11}, \Re_{21}$, and $L_{21}$.

$\begin{array}{ll}N_{2}=1 & \begin{array}{l}\text { number of turns in each shading ring } \\ u\end{array} \\ i_{1} & \begin{array}{l}\text { ac voltage applied to the terminals of the main } \\ \text { coil }\end{array} \\ i_{2} & \text { current through the main coil } \\ \phi_{1} & \text { magnetic flux through the main coil } \\ \phi_{2} / 2 & \text { magnetic flux through each shading ring } \\ S & \text { cross-section of the magnetic core } \\ \Re & \text { reluctance of the magnetic circuit }\end{array}$

To model their effects, the two identical shading rings should be coils with a single turn, where $N_{2}=1$. Since the EE core of the contactor is symmetrical to the central pole, the magnetic circuit of Fig. 1(b) can be redrawn as shown in Fig. 1(c). The two exterior legs and their respective shading rings in Fig. 1(b) were reduced to a unique equivalent leg [10].

The reluctances of the equivalent magnetic circuit described in Fig. 1(b) can be expressed as follows:

$$
\Re=\frac{l_{m}}{\mu_{0} \mu_{r} S_{m}}+\frac{l_{f}-e}{\mu_{0} \mu_{r} S_{f}}+\frac{x+e}{\mu_{0}\left(S_{f}+S_{m}\right) / 2} .
$$

Let $\Re_{1 a}$ and $\Re_{1 b}$ be the reluctance of the portion of pole 1 (length $l_{3}$ ) that contains the shading ring and the reluctance of the portion that does not contain it, as shown in Fig. 1

$$
\begin{aligned}
\Re_{1 a} & =\frac{l_{3}}{\mu_{0} \mu_{r} S_{f 1 a}}+\frac{x}{\mu_{0} S_{f 1 a}} \\
\Re_{1 b} & =\frac{l_{3}}{\mu_{0} \mu_{r} S_{f 1 b}}+\frac{x}{\mu_{0} S_{f 1 b}} .
\end{aligned}
$$

The equivalent reluctance that is a result of the parallel between $\Re_{1 a}$ and $\Re_{1 b}$ is

$$
\Re_{1}=\frac{\Re_{1 a} \Re_{1 b}}{\Re_{1 a}+\Re_{1 b}}=\frac{l_{3}}{\mu_{0} \mu_{r}\left(S_{f 1 a}+S_{f 1 b}\right)}+\frac{x}{\mu_{0}\left(S_{f 1 a}+S_{f 1 b}\right)} .
$$

The reluctance $\Re_{2}$ results in

$$
\Re_{2}=\frac{l_{m 1}}{\mu_{0} \mu_{r} S_{m 1}}+\frac{0.5 l_{m 2}}{\mu_{0} \mu_{r} S_{m 2}}+\frac{0.5 l_{f 2}}{\mu_{0} \mu_{r} S_{f 2}}+\frac{l_{f}-l_{3}}{\mu_{0} \mu_{r} S_{f 1}} .
$$

\section{A. Calculation of $L_{11}, \Re_{11}, L_{21}=L_{12}$, and $\Re_{12}=\Re_{21}$}

In the calculation of auto-inductance $L_{11}$ and reluctance $\Re_{11}$, only the effects of the main coil must be considered. Therefore, the effects of the shading rings must be eliminated, giving the equivalent circuit shown in Fig. 2.

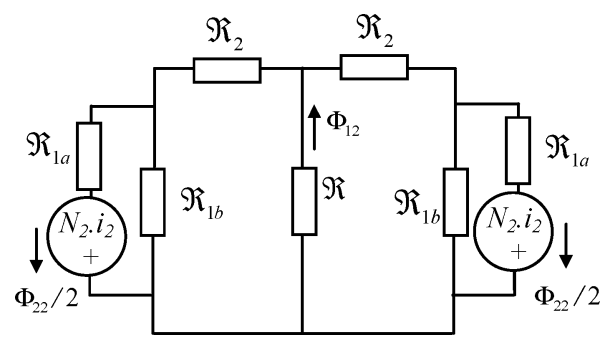

Fig. 3. Equivalent magnetic circuit for calculating $\Phi_{22}, L_{22}, \Phi_{12}$ and $L_{12}$.

From Fig. 2, the magnetomotive force $\Im_{1}$ is derived as

$$
\Im_{1}=N_{1} i_{1}=\Phi_{11}\left(\Re+\Re_{1} / 2+\Re_{2} / 2\right) .
$$

From (6), the flux $\Phi_{11}$ can be written as

$$
\Phi_{11}=\frac{N_{1} i_{1}}{\Re+\Re_{1} / 2+\Re_{2} / 2} .
$$

The auto-inductance $L_{11}$ and the reluctance $\Re_{11}$ can then be expressed as follows:

$$
L_{11}=N_{1}^{2} \frac{\Phi_{11}}{\Im_{1}}=\frac{N_{1}^{2}}{\Re+\Re_{1} / 2+\Re_{2} / 2}=\frac{N_{1}^{2}}{\Re_{11}} .
$$

From Fig. 2, the flux $\Phi_{21}$ that the main coil induces in the equivalent shading ring is computed as

$$
\Phi_{21}=\Phi_{11} \frac{\frac{\Re_{1 b}}{2}}{\frac{\Re_{1 a}}{2}+\frac{\Re_{1 b}}{2}}=\frac{N_{1} i_{1}}{\left(\Re+\frac{\Re_{2}}{2}+\frac{\Re_{1}}{2}\right)\left(\frac{\Re_{1 a}+\Re_{1 b}}{\Re_{1 b}}\right)} .
$$

From (9), the expressions of inductance $L_{21}$ and reluctance $\Re_{21}$ are obtained as

$$
L_{21}=\frac{N_{1} N_{2}}{\left(\Re+\frac{\Re_{2}}{2}\right)\left(1+\frac{S_{f 1 b}}{S_{f 1 a}}\right)+\frac{\Re_{1 a}}{2}}=\frac{N_{1} N_{2}}{\Re_{21}} .
$$

\section{B. Calculation of $L_{22}$ and $\Re_{22}$}

To obtain the expressions of the auto-inductance and the reluctance that is due only to the two shading rings ( $L_{22}$ and $\Re_{22}$ ), the effects of the main coil must be eliminated, which results in the equivalent circuit shown in Fig. 3.

Assuming that $\Re^{\prime}=\Re+\left(\Re_{2} / 2\right)$ gives

$$
\Im_{2}=N_{2} i_{2}=\Phi_{22}\left(\frac{\Re_{1 a}}{2}+\frac{\frac{\Re_{1 b}}{2} \Re^{\prime}}{\frac{\Re_{1 b}}{2}+\Re^{\prime}}\right)
$$

the expression of $\Phi_{22}$ can be derived.

$$
\Phi_{22}=\frac{N_{2} i_{2}}{\frac{\Re_{1 a}}{2}+\frac{\frac{\Re_{1 b}}{2} \Re^{\prime}}{\frac{\Re_{1 b}}{2}+\Re^{\prime}}} .
$$

Equation (12) leads to

$$
L_{22}=N_{2}^{2} \frac{\Phi_{22}}{f m m_{2}}=\frac{N_{2}^{2}}{\frac{\Re_{1 a}}{2}+\frac{\Re_{1 b} \Re^{\prime}}{\Re_{1 b}+2 \Re^{\prime}}}=\frac{N_{2}^{2}}{\Re_{22}} .
$$

Given that the reluctance depends on the magnetic permeability value, the latter must be computed. The magnetic core has a nonlinear $B-H$ characteristic. This nonlinearity means that the value of relative permeability $\mu_{r}$ is not constant because 
it is a function of the strength of the magnetic field. In order to determine the relationship between fields $B$ and $H$, the first magnetization curve of the magnetic core was obtained experimentally, and then adjusted by means of the Froëlich equation [11]

$$
B=\frac{a H}{1+b H} .
$$

From (14), the values of $\mu$ can be computed as

$$
\mu=\mu_{0} \cdot \mu_{r}=a-b B=a-b \Phi / A
$$

where $A$ and $\Phi$ are respectively the area and the flux through the cross-section of the magnetic core when the permeability is computed. Parameters $a$ and $b$ in (14) were adjusted using the least-squares optimization algorithm, giving the values $a=$ 0.0010 and $b=0.00043$. These parameters are given to students so that they can complete the practical session.

\section{DYNAMICS OF THE AC CONTACTOR}

In this section, the forces that govern the closure of the contactor are analyzed. They are the basis for establishing the dynamic equations of the ac contactor.

\section{A. Magnetic Force}

In the absence of magnetic saturation, the stored magnetic energy is equal to the magnetic co-energy. Under this assumption, the magnetic energy $W_{\text {mag }}$ that the coils of Fig. 1(a) supply to the magnetic core can be expressed as follows:

$$
W_{\text {mag }}=\frac{1}{2} L_{11} i_{1}^{2}+\frac{1}{2} L_{22} i_{2}^{2}+L_{12} i_{1} i_{2} .
$$

The differential equations that govern the dynamics of the ac contactor are solved by applying the ode 45 numerical method, which is based on the Dormand-Prince pair, a Runge-Kutta (4), (5) formula. Although the contactor and its magnetic circuit are a nonlinear system, by choosing a small enough integration step-size, one can expect it to behave in a linear fashion during the transition between two consecutive states because they are very close in time. By applying the explained procedure, the final result will reproduce the nonlinear behavior of the system, as confirmed by the simulated results shown in Section IX (Fig. 7), where after the closure, the current shows a clearly nonsinusoidal waveform due to the saturation of the magnetic core.

The magnetic energy is expressed in terms of the reluctances. Hence, (16) can be expressed as

$$
W_{\text {mag }}=\frac{1}{2} \Re_{11} \Phi_{11}^{2}+\frac{1}{2} \Re_{22} \Phi_{22}^{2}+\Re_{12} \Phi_{12} \Phi_{21} .
$$

The magnetic force can be calculated from the magnetic energy stored by the system, as

$$
F_{\mathrm{mag}}=-\frac{d W_{\mathrm{mag}}}{d x} .
$$

By computing the position of the movable core $x$ and the magnetic energy $W_{\text {mag }}$ for each instant of time, the magnetic force can be computed as the numerical derivative of $W_{\text {mag }}$ with respect to $x$.

\section{B. Dynamics of the Movable Core}

The forces involved in the movement of the moving core are the magnetic force, the friction force, and the forces generated by the springs (the return spring and the three phase springs).

The force produced by the return spring is opposed to the magnetic force during closure, and can be expressed as

$$
F_{k_{1}}=k_{1}\left(d_{A_{-} 0}+d_{A}-x\right) .
$$

The force produced by the three phase springs (the contactor dealt with has three power contacts) must be taken into account only when $x<d_{A}-d_{C}$ (the impact between the movable and the fixed contacts takes place when $x=d_{A}-d_{C}$ ). Therefore,

$\begin{cases}F_{3 k_{2}}=0, & \text { when } x \geq \mathrm{d}_{\mathrm{A}}-\mathrm{d}_{\mathrm{C}} \\ F_{3 k_{2}}=3 k_{2} & \\ \quad \times\left[d_{C \_0}+d_{A}-d_{C}-\left(x-x_{C}\right)\right], & \text { when } x<\mathrm{d}_{\mathrm{A}}-\mathrm{d}_{\mathrm{C}} .\end{cases}$

The friction force is supposed to be proportional to the speed of the movable core and can be expressed as

$$
F_{f}=-b \dot{x} .
$$

The impact between the movable and the fixed cores is assumed to be nonelastic. To analyze this collision, it is necessary to deal with the restitution coefficient $\varepsilon \in[0,1]$, which is defined as

$$
\varepsilon=-\dot{x}_{f} / \dot{x}_{i}
$$

where $\dot{x}_{i}$ and $\dot{x}_{f}$ are respectively the velocities of the movable core just before and just after the impact. Taking into account all the forces involved in the dynamics of the movable core, the result of applying the second Newton law is

$$
\begin{aligned}
& \ddot{x}=\frac{k_{1}}{m}\left(d_{A_{-} 0}+d_{A}-x\right)+\underbrace{\frac{3 k_{2}}{m}\left[d_{C \_0}+d_{A}-d_{C}-\left(x-x_{C}\right)\right.}_{\text {when } x<d_{A}-d_{C}}] \\
& -\frac{1}{m}\left|-\frac{\partial}{\partial x}\left[\frac{1}{2} \Re_{11} \Phi_{11}^{2}+\frac{1}{2} \Re_{22} \Phi_{22}^{2}+\Re_{12} \Phi_{12} \Phi_{21}\right]\right|-\frac{b}{m} \dot{x}
\end{aligned}
$$

where

$$
m= \begin{cases}m_{A}+3 m_{C}, & \text { when } x \geq d_{A}-d_{C} \\ m_{A}, & \text { when } x<d_{A}-d_{C} .\end{cases}
$$

\section{Dynamics of the Movable Contacts}

When the movable core attains the position $x=d_{A}-d_{C}$, the movable contacts collide with the fixed contacts, and the three phase springs start to become compressed.

When $x \geq d_{A}-d_{C}$, the phase springs are not compressed and the following are fulfilled:

$$
\left\{\begin{array}{l}
F_{3 k_{2}}=0 \\
\ddot{x}_{C}=\ddot{x} \\
x_{C}=x-\left(d_{A}-d_{C}\right) .
\end{array}\right.
$$


When $x<d_{A}-d_{C}$, the phase springs are compressed, and the following are fulfilled:

$$
\left\{\begin{array}{l}
F_{3 k_{2}}=3 k_{2}\left[d_{C \_0}+d_{A}-d_{C}-\left(x-x_{C}\right)\right] \\
\ddot{x}_{C}=\ddot{x}-3 k_{2}\left(d_{C \_0}+x_{C}\right) /\left(3 \cdot m_{C}\right) .
\end{array}\right.
$$

Thus, the relationship between the acceleration of the movable contacts $\ddot{x}_{C}$ and the movable core $\ddot{x}_{A}$ is given by

$$
\left\{\begin{array}{l}
\ddot{x}_{C}=\ddot{x}+\ddot{x}_{C}^{A} \\
\ddot{x}_{C}^{A}=F_{3 k_{2}} / m_{C}=-3 k_{2}\left(d_{C \_0}+x_{C}\right) /\left(3 m_{C}\right)
\end{array}\right.
$$

where $\ddot{x}_{C}^{A}$ is the acceleration of the movable contacts with respect to the system of reference fixed in the movable core.

\section{Electrical and Magnetic Equations OF THE AC CONTACTOR}

From Fig. 1(b) and (c), the following can be derived:

$$
\left.\begin{array}{l}
N_{1} i_{1}=\Phi_{1}\left(\Re^{\prime}+\frac{\Re_{1 b}}{2}\right)-\Phi_{2} \frac{\Re_{1 b}}{2} \\
N_{2} i_{2}=-\Phi_{1} \frac{\Re_{1 b}}{2}+\Phi_{2}\left(\frac{\Re_{1 a}}{2}+\frac{\Re_{1 b}}{2}\right)
\end{array}\right\} .
$$

The electrical equations of the main coil and the shading rings are given as

$$
\left.\begin{array}{l}
u=R_{1} i_{1}+N_{1} \frac{d \Phi_{1}}{d t} \\
0=2 R_{2} i_{2}+N_{2} \frac{d \Phi_{2}}{d t}
\end{array}\right\}
$$

where

$$
\Phi_{1}=\Phi_{11}+\Phi_{12} \text { and } \Phi_{2}=\Phi_{22}+\Phi_{21} .
$$

Magnetic and electrical equations (28)-(30) are coupled with dynamic equations (23)-(27). Thus, to describe the dynamic behavior of the contactor, coupled equations (23)-(30) must be solved simultaneously.

\section{Corrections Due to FluX Fringing}

When dealing with large air gaps, flux fringing must be taken into account. Flux fringing describes the spreading of flux lines in an air gap from the shortest path between poles in a magnetic circuit. The spreading of the flux lines increases the effective cross-section of the air gap, which in turn increases the inductance of the air gap and decreases its reluctance. The effects of flux fringing become more evident as the air gap becomes larger, increasing the effective area of the air gap. Thus, to consider the effects of flux fringing, the area of the cross-section of the air gap should be multiplied by a correction factor $F \geq 1$ [12], [13]. The fringing factor $F$ can be calculated as

$$
F=\left\{\begin{array}{ll}
1+\frac{x}{S^{1 / 2}} \ln \left(\frac{2 G}{x}\right), & \text { if } x>0 \\
1, & \text { if } x=0
\end{array} .\right.
$$

where $x$ is the air gap, $S$ is the area of the cross-section of the poles, and $G$ is the winding length. Equation (31) can be applied when one is dealing with UU or EE-cores, the latter being the object of study in this case. Thus, the fringing factor obtained from (31) must be introduced into (1)-(4).

Fig. 4 shows the effects of flux fringing. As depicted in Fig. 4, the effective area of the air gap increases because of the effect

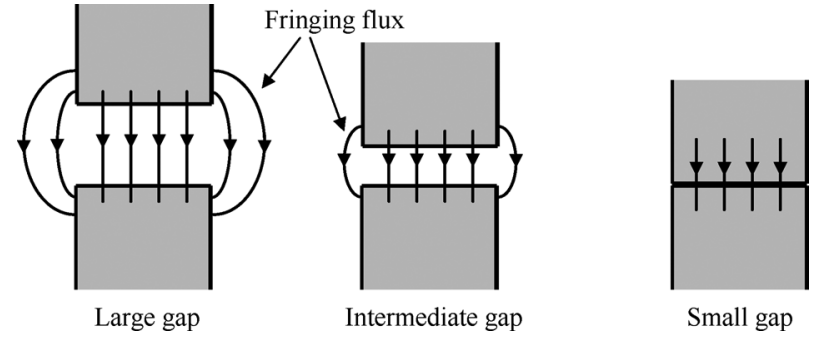

Fig. 4. Fringing flux paths as a function of the air gap.

of the fringing flux paths, which alters the reluctance of the air gap.

\section{SIMULINK MODEL}

The equations given in Sections III-VI must be implemented in Simulink to simulate the dynamic behavior of the contactor. Students are encouraged to separate the problem into two clearly differentiated blocks: the mechanical and the electromagnetic block. The mechanical block is composed of two subblocks: one that calculates the kinematics of the movable core and one that calculates the kinematics of the movable contacts. Whereas the mechanical block solves the dynamic equations shown in Section IV, the electromagnetic block solves the equations shown in Sections III, V, and VI. The mechanical block calculates the position, velocity, and acceleration of both the movable core and the contacts. As input, it needs the magnetic force that is the output of the electromagnetic block. The electromagnetic block receives as inputs the line voltage and the position of the movable core (which the mechanical block gives as output). Thus, the mechanical and the electromagnetic blocks are coupled because the output of one is the input of the other and vice versa.

\section{EFFECTS OF THE SHADING RINGS}

A shading ring is a metal ring, usually made of copper, that is placed around a portion of the section of the magnetic core by means of a notch at its end. Shading rings play a very important role in the dynamics of ac-powered contactors, working as shorted secondary coils in the electromagnet, the primary coil being the main coil connected to the power supply. dc-powered coils generate constant magnetic fields under stationary conditions that generate an attractive magnetic force. Thus, dc-powered contactors do not require shading rings. In contrast, ac-powered coils generate sinusoidal magnetic fields that produce a force that drops to zero twice in each cycle $(50 / 60 \mathrm{~Hz})$. This effect creates unwanted chatter or vibration of the contacts, which can be eliminated by placing a shading ring in the contactor core. The current flowing through the shading ring generates a magnetic field that is out of phase with the one generated by the contactor coil. This setup produces a force that does not drop to zero, thus eliminating the vibrations.

To study the effects of the shading rings, it is helpful to study a magnetic circuit in which the main coil is in an open circuit 


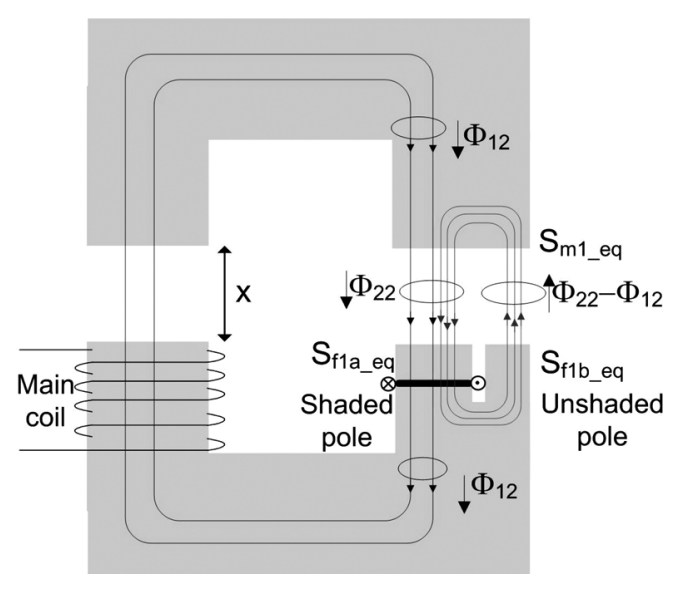

Fig. 5. Plot of the flux through the central leg of the contactor when the effects of the main coil have been eliminated.

state, thus eliminating its effects. Fig. 5 shows how the shading rings work.

As Fig. 5 shows, the magnetic flux generated by the equivalent shading ring has two paths. A portion of it, $\left(\Phi_{22}-\Phi_{12}\right)$, loops through $\mathrm{S}_{\mathrm{f} 1 \mathrm{a} \_\mathrm{eq}}$ and $\mathrm{S}_{\mathrm{f} 1 \mathrm{~b} \_\mathrm{eq}}$. Another portion, $\left(\Phi_{12}\right)$, circulates through the main coil. Thus, there is a flux loop between $\mathrm{S}_{\mathrm{fla} \_\mathrm{eq}}$ and $\mathrm{S}_{\mathrm{f} 1 \mathrm{~b} \_\mathrm{eq}}$, which results in $\Phi_{12}-\Phi_{22}$ and $\Phi_{22}$ being out of phase. The relation between the phase angles of these two fluxes is essential for eliminating the chattering of the contacts.

The main coil and the shading ring acts in a similar way to a transformer. The current induced in the shading ring is a result of the time-varying magnetic field created by the main coil. Ideally, when this magnetic field is in the zero crossings of the sine wave, the strength of the current induced in the shading ring is at its peak. Similarly, when the strength of the main coil's magnetic field is at its peak the current in the shading coil is zero. Thus, the alternating magnetic field created by the shading ring is ideally shifted $90^{\circ}$ out of phase by the magnetic field created by the primary coil.

The portion of the magnetic core without the shading ring has a magnetic field that is generated by the main coil of the contactor, and the portion that contains the shading ring has a magnetic field that is out of phase with the former one. The result is a magnetic field that is never zero, in both portions of the magnetic core simultaneously.

\section{EXPERIMENTAL RESULTS AND SIMULATIONS}

A fundamental aim of this practical is to introduce students to the topic of modeling, which involves model generation and model validation. In order to validate the parametric model of the contactor, students are encouraged to register voltage $u$ and current $i_{1}$ through the main coil of the contactor during a closing maneuver using a digital oscilloscope. The closure of the contactor is carried out by applying a $50-\mathrm{Hz}, 230-\mathrm{V}$ ac voltage. Data obtained from this experimental test is used to validate the model of the contactor. Students are provided with the electrical and mechanical parameters of the contactor they analyze.

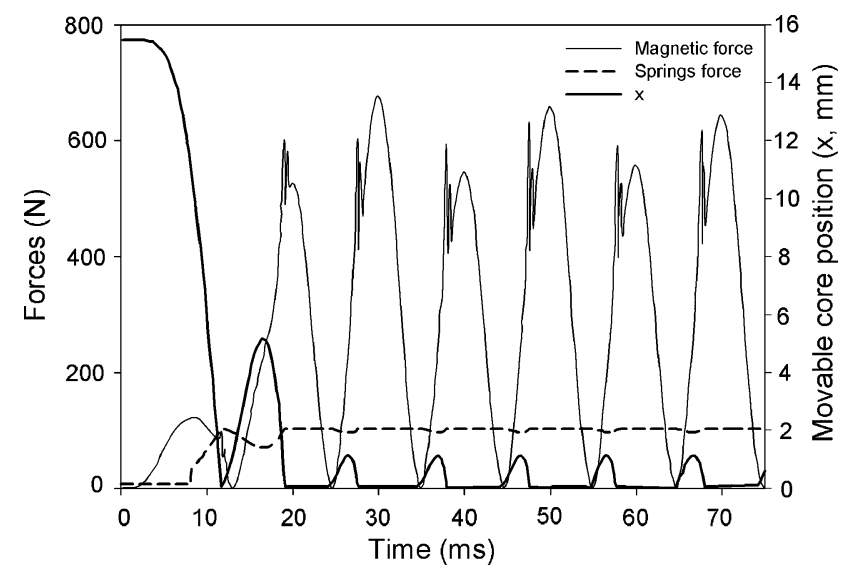

Fig. 6. Simulated magnetic and spring forces and the trajectory of the movable core position when the shading rings have been removed.

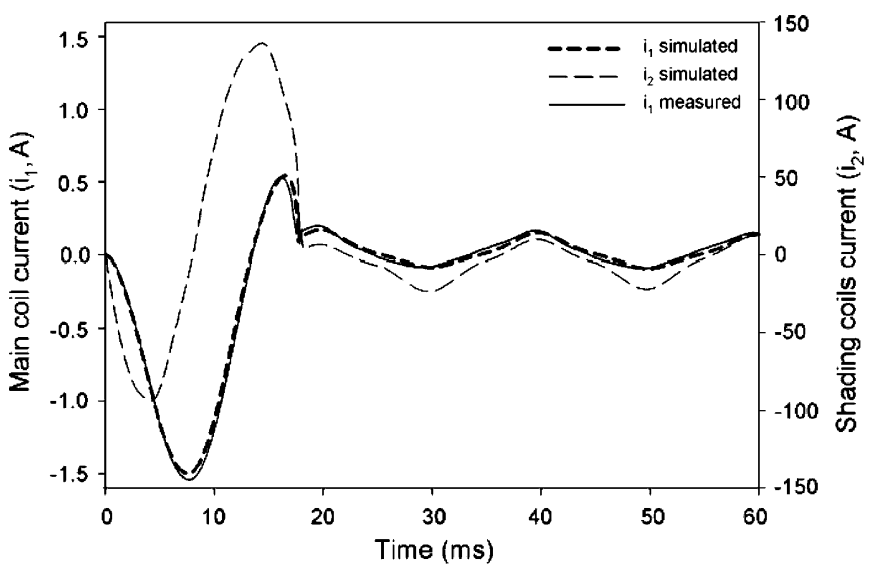

Fig. 7. Measured and simulated current through the main coil $\left(i_{1}\right)$ and simulated current through each shading ring $\left(i_{2}\right)$.

\section{A. AC Contactor Without Shading Rings}

As explained in Section VIII, by eliminating the effects of the shading rings the movable core is subjected to unwanted chattering. This effect was simulated as shown in Fig. 6.

Fig. 6 clearly shows the chattering of the movable core after closure, which is due to the magnetic force dropping to zero twice in each cycle $(50 \mathrm{~Hz})$. This result shows the students that shading rings are absolutely necessary.

\section{B. AC Contactor With Shading Rings}

Fig. 7 shows the measured and simulated current $i_{1}$ through the main coil of the contactor and the simulated values of the current through the shading rings (which is very difficult to measure) during closure of the contactor. Note that the maximum value of current $i_{2}$ is approximately 100 times greater than the maximum value of $i_{1}$.

Fig. 7 shows close agreement between the measured and the simulated currents. It is an experimental validation of the suitability of the contactor model proposed in this work. The closing action of the contactor-that is, the period during which the movable core is moving_lasts until approximately $18 \mathrm{~ms}$. From then on, the magnetic circuit remains closed and the magnetic circuit in essence behaves as a transformer. Thus, after closure, the currents $i_{1}$ and $i_{2}$ are almost in phase. From Fig. 7, it can be 


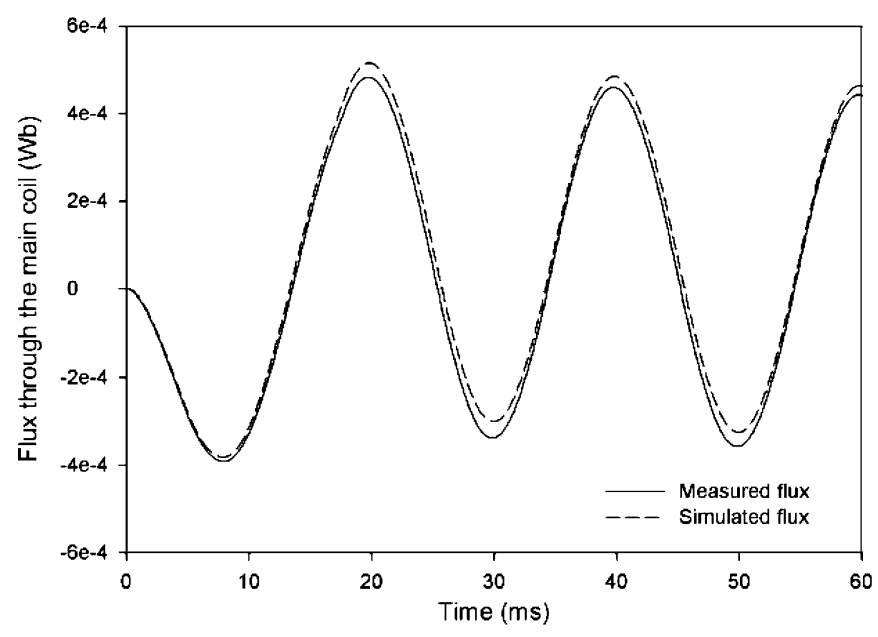

Fig. 8. Experimental and simulated flux through the main coil.

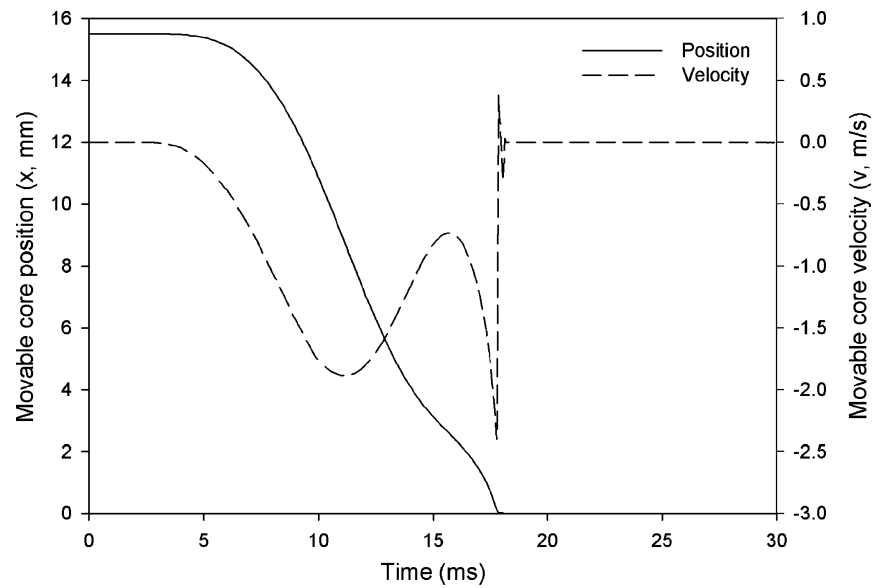

Fig. 9. Simulated profiles of the movable core position and velocity during closure.

deduced that the electrical transient still lasts and the currents $i_{1}$ and $i_{2}$ will be zero-centered once the electrical transient is finished.

Fig. 8 displays both the experimental and the simulated flux through the contactor core. Fig. 8 once again proves the suitability of the model dealt with in this work. The real flux $\Phi_{1}$ through the main coil can be computed from time acquisitions $i_{1}$ and $u$.

$$
\Phi_{1}=\Phi_{11}+\Phi_{12}=\frac{1}{N_{1}} \int_{0}^{t}\left(u-i_{1} R_{1}\right) \cdot d t .
$$

Fig. 9 shows a plot of the simulated values of the position and the velocity of the movable core during closure. It can be observed that the closure of the movable core is produced at approximately $t=18 \mathrm{~ms}$. Note that in this case there is no chattering. Measuring the movable core position requires a noninvasive measuring device such as a sophisticated laser Doppler vibrometer, which is not readily available.

Fig. 10 plots the simulated values of the self-inductances $L_{11}$ and $L_{22}$ during closure of the contactor.

Fig. 11 depicts the magnetic force and the total force of the springs during the closure maneuver. Note that, once again,

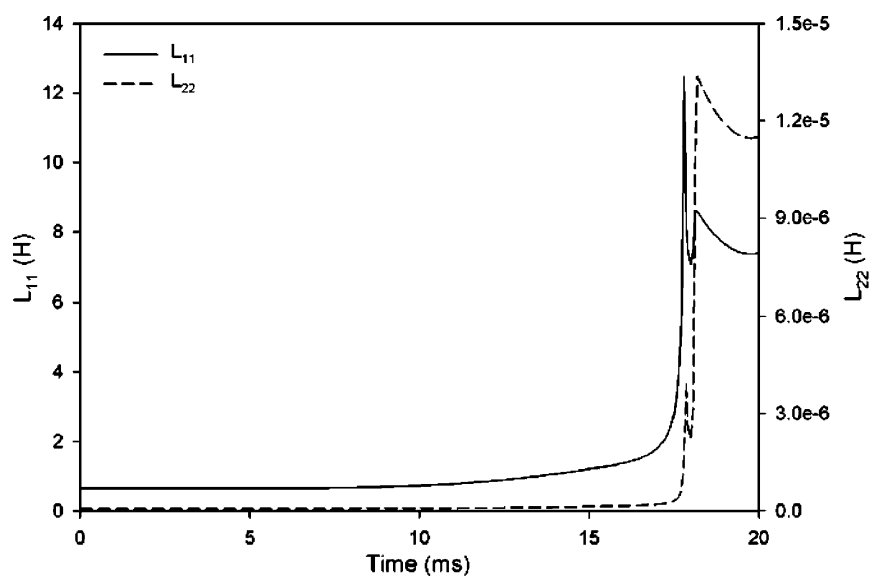

Fig. 10. Simulated profiles of the self-inductances $L_{11}$ and $L_{22}$ during closure of the contactor.

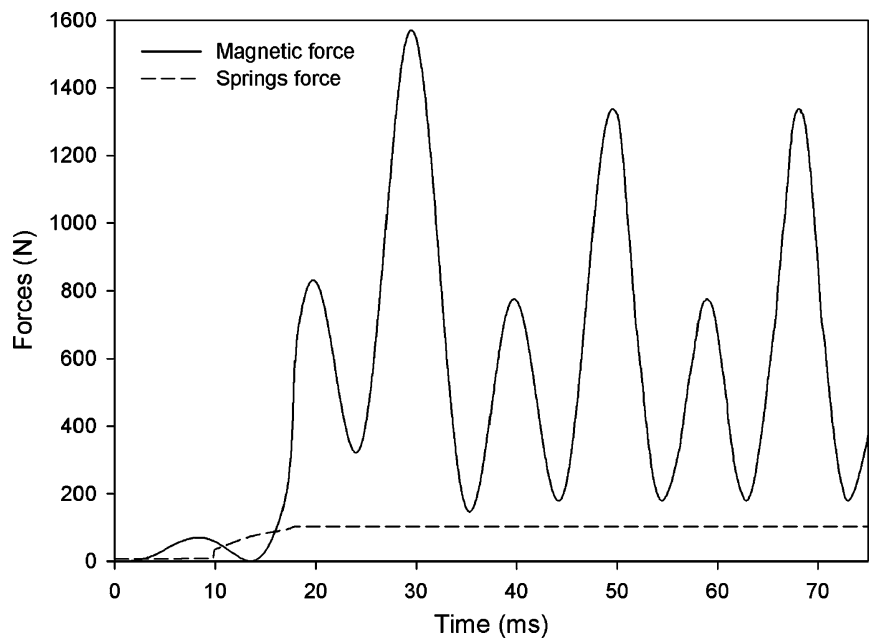

Fig. 11. Simulated values of the magnetic force and the total force of the return springs during a closure maneuver.

these forces are difficult to measure, but they are easily obtained from simulations. Fig. 11 clearly illustrates the effect of the shading rings, which consists in raising the magnetic force and avoiding the drop to zero, thus eliminating the vibrations of the movable armature.

\section{STUdent AsSESSMENT}

The proposed practical was taught to 75 undergraduate students at UPC as part of the Electrotechnology course in the second semester of the 2007-2008 academic year. The reactions of students to this practical were reasonably positive, although they said it increased their workload slightly.

The students were asked to fill out a questionnaire about the laboratory session, which proved to be an important source of feedback and allowed the instructors to identify possible errors and improvements. In the questionnaire, students were asked to assess various aspects related to their satisfaction with the proposed practical and its usefulness for consolidating their knowledge of the content studied. The questionnaire consisted of the six questions shown in Table I. The students had to grade their answers from 1 (strongly disagree) to 5 (strongly agree). 
TABLE I

QUESTIONNAIRE ANSWERED BY THE STUDENTS

\begin{tabular}{l|l}
\hline \multicolumn{1}{|c}{ Questions } & Score \\
\hline 1. I had previous knowledge of Matlab- & \\
Simulink. & \\
2. The help of the instructor was valuable. & \\
3. The level of the practical is appropriate. & \\
4. Teamwork has helped me in this & \\
practical. \\
5. This practical has helped me to \\
understand the theory better. \\
6. The content of this practical is valuable \\
for an engineer.
\end{tabular}

TABLE II

RESULT OF THE QUESTIONNAIRE ANSWERED BY THE STUDENTS

\begin{tabular}{|c|c|}
\cline { 2 - 2 } \multicolumn{1}{c|}{} & Average score \\
\hline Question 1 & 2.99 \\
Question 2 & 4.04 \\
Question 3 & 3.35 \\
Question 4 & 3.90 \\
Question 5 & 4.06 \\
Question 6 & 4.04 \\
\hline Total & 3.73 \\
\hline
\end{tabular}

Table II shows the average scores for each question. The answers to Question 1 indicate that the students had previously acquired knowledge of Matlab-Simulink to an intermediate level. Question 2 asks about the role of the instructors. The answers to this question clearly indicate that the guidance of the instructor is critical if the course objectives are to be met. As the scores for Question 3 show, most of the students felt the practical was suited to their level, although some felt that they had to put in considerable effort. Question 4 refers to the influence of teamwork on the final result of the practical. The results of this question show that the majority of the students think that teamwork is advantageous. The answers to Question 5 indicate that the methodology applied helps to consolidate the students' understanding of the theory related to this practical. Finally, Question 6 shows that students believe that the proposed methodology is useful for future engineers. Fig. 12 shows the overall results of the student questionnaire.

The average answer for all the questions was 3.73. This overall mark indicates a satisfactory degree of approval of the students of the methodology presented in this work. The conclusion is that the practical session was well regarded by the students and served to motivate them. It also helped them to gain a better understanding of the theoretical concepts involved in the behavior of ac-powered contactors.

\section{CONCLUSION}

The aim of this paper is to present a methodology for improving teaching effectiveness by introducing students to the topic of simulating a real device such as an ac-powered contactor. The behavior of the shading rings is not easy for students to understand, and this practical contributes to a better understanding. Dynamic simulation is a salient topic in many re-

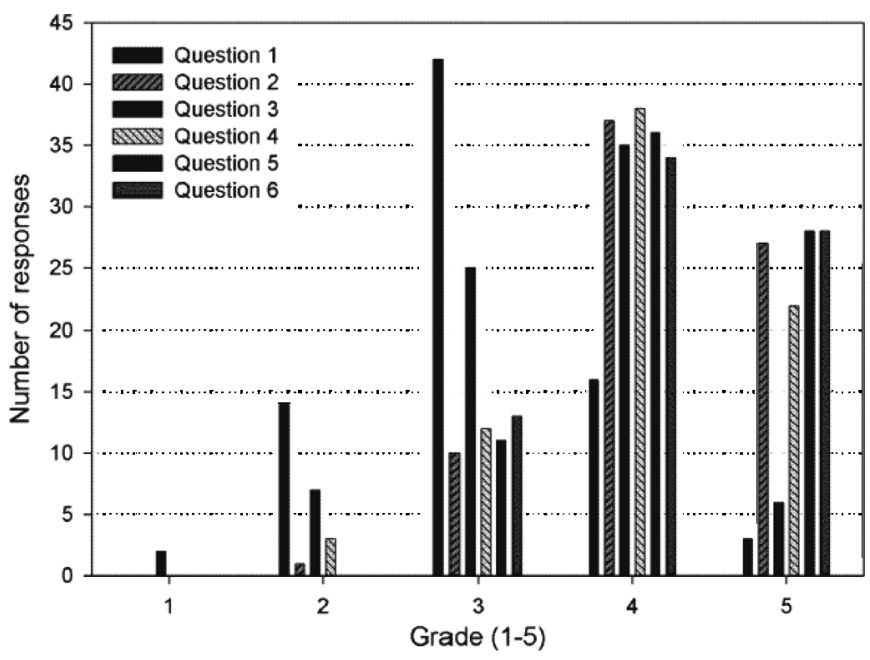

Fig. 12. Answers to the questionnaire.

search areas - such as ergonomics, chemistry, and mechanical engineering - that allows better understanding of the transient and steady-state response of the systems under study. Due to its growing relevance, graduates in electrical engineering must be competent in this area.

The methodology proposed in this work was applied in two laboratory sessions lasting $2 \mathrm{~h}$ each. Students are grouped into teams of three. First, the voltage and the current during the closure of the contactor are measured. Afterward, students set out the electrical, magnetic, and mechanical equations that govern the dynamic behavior of the ac contactor and then simulate the real device. Second, from the simulation results, students visualize the evolution over time of the mechanical and magnetic variables that are very difficult to measure experimentally. In these laboratory sessions, students also learn how to validate a simulation model by measuring some available data of the real device (in the example, these data are the voltage and the current through the main coil) and by comparing measured data with data resulting from simulations. Additionally, this practical session has various aspects that are of pedagogic interest:

- It improves teaching effectiveness.

- It fosters student motivation (modeling of a real device).

- It introduces the students to the topic of validating the model by comparing experimental data with data resulting from simulations.

- It provides the students with practical skills such as those involved in teamwork, problem-based learning, problem solving, and case-study analysis.

- Students study, implement, and solve the electromechanical coupled equations related to the dynamic behavior of the ac-powered contactor.

\section{ACKNOWLEDGMENT}

In this work, the CL08A300M contactor manufactured by GE Industrial Systems, which was fed by an LB4AN coil, was used.

\section{REFERENCES}

[1] M. Wada, H. Yoshimoto, and Y. Kitaide, "Dynamic analysis and simulation of electromagnetic contactors with AC solenoids," in Proc. IEEE 28th Annu. IECON, 2002, vol. 4, pp. 2745-2751. 
[2] Z. Wróblewski, "A method of gamma-beta durability determination for reliability tests of brand-new AC electromagnetic contactors," Eur. T. Electr. Power, vol. 10, no. 4, pp. 219-224, Jul./Aug. 2000.

[3] Z. Wróblewski, "Digital simulation of reliability of contacts used in AC electromagnetic contactors," Eur. T. Electr. Power, vol. 8, no. 3, pp. 201-206, May/Jun. 1998.

[4] S. Karmalkar, "Introducing the Device Modeling Procedure to Electrical Engineering Students," IEEE Trans. Educ., vol. 50, no. 2, pp. 137-142, May 2007.

[5] M. J. Durán, S. Gallardo, S. L. Toral Rocío Martínez-Torres, and F. J. Barrero, "A learning methodology using Matlab/Simulink for undergraduate electrical engineering courses attending to learner satisfaction outcomes," Int. J. Technol. Des. Educ., vol. 17, no. 1, pp. 55-73, Jan. 2007.

[6] S. Ayasun and C. O. Nwankpa, "Transformer tests using MATLAB/ Simulink and their integration into undergraduate electric machinery courses," Comput. Appl. Eng. Educ., vol. 14, no. 2, pp. 142-150, Jul. 2006.

[7] M.-C. Tsai, C.-C. Chou, and M.-F. Hsieh, "Development of a realtime servo control test bench," IEEE Trans. Educ., vol. 40, no. 4, pp. 242-252, Nov. 1997.

[8] S. Ayasun and C. O. Nwankpa, "Induction motor tests using MATLAB/ Simulink and their integration into undergraduate electric machinery courses," IEEE Trans. Educ., vol. 48, no. 1, pp. 37-46, Feb. 2005.

[9] A. A. Rodriguez, R. P. Metzger, O. Cifdaloz, and T. Dhirasakdanon, "Description of a Modeling, Simulation, Animation, and Real-Time Control (MoSART) environment for a class of electromechanical systems," IEEE Trans. Educ., vol. 48, no. 3, pp. 359-374, Aug. 2005.

[10] J.-R. Riba Ruiz and A. García Espinosa, "A novel parametric model for AC contactors," IEEE Trans. Magn., vol. 44, no. 9, pp. 2215-2218, Sep. 2008.

[11] A. García Espinosa, J.-R. Riba Ruiz, and X. A. Morera, "A sensorless method tor controlling the closure of a contactor," IEEE Trans. Magn., vol. 43, no. 10, pp. 3896-3903, Oct. 2007.

[12] O. Dezuari, S. E. Gilbert, E. Belloy, and M. A. M. Gijs, "Development of a novel printed circuit board technology for inductive device applications," Sensor. Actuat. A-Phys., vol. 76, no. 1-3, pp. 349-355, 1999.

[13] A. Van den Bossche, V. Valchev, and T. Filchev, "Improved approximation for fringing permeances in gapped inductors," in Proc. Industry Appl. Conf. (37th IAS Annu. Meeting), 2002, vol. 2, pp. 932-938.
Jordi-Roger Riba Ruiz received the M.S. degree in physics and the Ph.D. degree from the Universitat de Barcelona, Barcelona, Spain, in 1990 and 2000, respectively.

In 1992, he joined the College of Industrial Engineering of Igualada, Universitat Politècnica de Catalunya, Barcelona, Spain, as a full-time Lecturer, and he joined the Electric Engineering Department in 2001. He belongs to the Motion and Industrial Control Group (MCIA). His research interests include electromagnetic devices modeling, signal processing methods, electric machines, variable-speed drive systems, and fault detection algorithms.

Antonio Garcia Espinosa (M'05) received the M.S. degree in electrical engineering and the Ph.D. degree from the Universitat Politècnica de Catalunya (UPC), Barcelona, Spain, in 2000 and 2005 respectively.

In 2000, he joined the Electric Engineering Department of the UPC, where he is currently a Lecturer. He belongs to the Motion and Industrial Control Group (MCIA). His research interests include electromagnetic devices, electric machines, variable-speed drive systems, and fault-detection algorithms.

Luis Romeral (M'98) received the M.S. degree in electrical engineering and the Ph.D. degree from the Universitat Politècnica de Catalunya (UPC), Barcelona, Spain, in 1985 and 1995, respectively.

In 1988, he joined the Electronic Engineering Department, UPC, where he is currently an Associate Professor and the Director of the Motion and Industrial Control Group (MCIA), whose major research activities concern induction and permanent magnet motor drives, enhanced efficiency drives, fault detection and diagnosis of electrical motor drives, and improvement of educational tools. He has developed and taught post-graduate courses on programmable logic controllers, electrical drives and motion control, and sensors and actuators.

Dr. Romeral is a Member of the European Power Electronics and Drives Association and the International Federation of Automatic Control. 